Portland State University

PDXScholar

Electrical and Computer Engineering Faculty

Publications and Presentations

Electrical and Computer Engineering

$1-1-1985$

\title{
Spontaneous coherent pulsations in ring-laser oscillators
}

Lee W. Casperson

Portland State University

Follow this and additional works at: https://pdxscholar.library.pdx.edu/ece_fac

Part of the Electrical and Computer Engineering Commons

Let us know how access to this document benefits you.

\section{Citation Details}

Lee W. Casperson, "Spontaneous coherent pulsations in ring-laser oscillators," J. Opt. Soc. Am. B 2, 62-72 (1985).

This Article is brought to you for free and open access. It has been accepted for inclusion in Electrical and Computer Engineering Faculty Publications and Presentations by an authorized administrator of PDXScholar. Please contact us if we can make this document more accessible: pdxscholar@pdx.edu. 


\title{
Spontaneous coherent pulsations in ring-laser oscillators
}

\author{
Lee W. Casperson* \\ Department of Electrical Engineering, Portland State University, Portland, Oregon 97207
}

Received June 25, 1984; accepted August 27, 1984

\begin{abstract}
Complex spontaneous-pulsation phenomena have been reported in xenon lasers operating at $3.51-\mu \mathrm{m}$ wavelength, and a theoretical explanation for these effects has been given. However, the published models contain various simplifications and have so far yielded only qualitative agreement with xenon-laser experiments. Reported here is a more rigorous analytical model, which for the first time provides quantitative agreement with experimental observations. This model should also find direct application in interpreting or predicting the behavior of other spontaneously pulsing laser systems.
\end{abstract}

\section{INTRODUCTION}

In a laser with time-independent pumping and cavity loss rates, it is usually expected that a cw output beam will be produced. There are, however, many exceptions to this rule, and accounting for the experimentally observed instabilities has for many years been an active area of research. One of the first explanations suggested in investigations of laser instabilities was that the underlying equations are themselves unstable. This idea was proposed by Gurtovnik in 1958 in connection with transient effects in homogeneously broadened masers. ${ }^{1}$ He discovered that there is the possibility of a basic coherent instability in the semiclassical maser equations. A few years later, these results were extended and applied to the pulsations observed in ruby lasers by Uspenskii, Korobkin, Grasyuk, and Oraevskii. ${ }^{2-6}$ However, it was recognized, by the Russian authors at least, that the conditions for obtaining spontaneous coherent pulsations in single-mode homogeneously broadened lasers might be too severe to be satisfied in most practical lasers. ${ }^{7}$ Nevertheless, theoretical research on this subject has continued up to the present, and a more detailed historical overview is given in Ref. 8.

In spite of the negative theoretical results concerning spontaneous coherent pulsations in homogeneously broadened lasers, experimentalists have continued over the years to turn up simple laser systems that pulse spontaneously. In many cases, the cause of the pulsations has been difficult to determine or has never been discovered. The most important lasers in this context are the xenon and helium-xenon systems. Some time ago, this author discovered a curious pulsation instability that can afflict an otherwise well-behaved xenon laser. ${ }^{9-11}$ The instability sometimes manifests itself by the output of the laser being in the form of a highly periodic pulse train. Under other conditions, the pulsations become much more noiselike in character, and the transition from uniform pulsations to chaotic pulsations may involve a period-doubling effect. More recently, higher-order period-multiplying transitions have also been reported. ${ }^{12}$ Even when periodic pulsations are observed, the individual pulses often have a complex structure consisting of a dominant leading pulse followed by smaller echoes. At the time of the first experiments, no model was available that could account for this wealth of curious phenomena, and it was known that with ordinary rate-equation models no undamped pulsations should occur at all. ${ }^{10,13-16}$

Eventually, a semiclassical model was developed that was able to account in a qualitative way for several of the observed effects. ${ }^{11}$ This model includes independent lifetimes for the field in the cavity, the polarization, and the upper and lower laser levels, as well as an arbitrary level of Doppler broadening. The significance of the work was that it showed for the first time that the Lamb equations for an inhomogeneously broadened laser possess a low threshold instability. With reasonable values for the various parameters, the model predicts undamped pulsations of about the same frequency as that observed in the xenon-laser experiments. For both the standing-wave and traveling-wave lasers, the pulses are followed by damped trains of echo pulses. This theoretical echo behavior shows some resemblance to the xenon experiments mentioned above, but the quantitative discrepancies are substantial enough to cast some doubts on the predictive ability of the model.

In spite of the previously mentioned reservations, it has been deemed worthwhile to attempt to extract from the theoretical model at least a semi-intuitive physical interpretation for the pulsation instability. One result of such studies is that it is helpful to interpret the pulsations in terms of a modesplitting effect. ${ }^{17,18}$ In this effect, a high level of dispersion can make possible the existence of several frequencies all having the same average wavelength. In the case of an inhomogeneously broadened laser, extremely strong dispersion may occur in the wings of the transition line and also for frequencies near the frequency of a saturating mode, and, as a consequence, the mode may split into several different frequencies. ${ }^{19}$

With the recent increased interest in the subject ${ }^{12,20-32}$ and the increase in high-quality published data concerning the instability in xenon, $12,20,24,28,31$ it becomes worthwhile to develop a theoretical model that can provide something better than the qualitative agreement with data that has been possible heretofore. The development of such a model constitutes most of the content of this paper. In Section 2, a Maxwell-Schrödinger semiclassical analysis is applied to a ring- 
laser oscillator. In this formulation, the model includes several improvements over our previous treatment. ${ }^{11}$ A purely mathematical improvement is that the electric-field amplitude is expressed in terms of its real and imaginary parts rather than its magnitude and phase. Although this modification sounds trivial, it eliminates some difficulties in the numerical integration procedures. There are also several physical improvements. For example, in our earlier model, it was assumed, following Lamb, ${ }^{33}$ that atoms in the upper laser level can decay spontaneously but only to someplace other than the lower laser level. In fact, however, most spontaneous decays of the upper laser level in xenon take place directly to the lower level, and, in the present model, an arbitrary branching ratio is possible. Another important improvement is the inclusion of the velocity-changing collisions that lead to spectral cross relaxation in Doppler-broadened lasers. A normalized version of the model is obtained in Section 3.

Before any meaningful transient solutions can be attempted, it is necessary to obtain physically realistic values for all the parameters involved. Included in Section 4 is a systematic discussion of these parameters, and an attempt is made to deduce the best values based on published studies of the xenon laser. Except with respect to spectral cross relaxation, all the necessary xenon-laser parameters can be determined with uncertainties of at most a few percent. Several representative solutions of the laser equations are discussed in Section 5, including the effects of detuning and pressure variations. Within their range of applicability, the equations seem to provide quantitative agreement with available experimental data. Other results of interest concern the increase in average power when pulsations occur and also the existence of many discrete regions of chaotic oscillation.

\section{THEORY}

The starting point for this analysis is a set of MaxwellSchrödinger equations that is similar to the set used in previous studies of spontaneous pulsations. As written here, these equations most closely resemble the starting set in Ref. 18, where inhomogeneous broadening owing to both the Doppler effect and to a distribution of intrinsic resonance frequencies has been included. Thus the equations for the time and space dependence of the density matrix are

$$
\begin{aligned}
& \left(\frac{\partial}{\partial t}+\frac{v \partial}{\partial z}\right) \rho_{a b}\left(v, \omega_{\alpha}, z, t\right)=-\left(i \omega_{\alpha}+\gamma\right) \rho_{a b}\left(v, \omega_{\alpha}, z, t\right) \\
& -\frac{i \mu}{\hbar} E(z, t)\left[\rho_{a a}\left(v, \omega_{\alpha}, z, t\right)-\rho_{b b}\left(v, \omega_{\alpha}, z, t\right)\right] \\
& \left(\frac{\partial}{\partial t}+\frac{v \partial}{\partial z}\right) \rho_{a a}\left(v, \omega_{\alpha}, z, t\right) \\
& =\lambda_{a}\left(v, \omega_{\alpha}, z, t\right)-\gamma_{a} \rho_{a a}\left(v, \omega_{\alpha}, z, t\right) \\
& +\left[\frac{i \mu}{\hbar} E(z, t) \rho_{b a}\left(v, \omega_{\alpha}, z, t\right)+c . c .\right] \\
& \quad-\int \Gamma_{a}\left(v^{\prime}, v\right) \rho_{a a}\left(v, \omega_{\alpha}, z, t\right) \mathrm{d} v^{\prime} \\
& \quad+\int \Gamma_{a}\left(v, v^{\prime}\right) \rho_{a a}\left(v^{\prime}, \omega_{\alpha}, z, t\right) \mathrm{d} v^{\prime}
\end{aligned}
$$

$$
\begin{aligned}
\left(\frac{\partial}{\partial t}+\right. & \left.\frac{v \partial}{\partial z}\right) \rho_{b b}\left(v, \omega_{\alpha}, z, t\right) \\
= & \lambda_{b}\left(v, \omega_{\alpha}, z, t\right)-\gamma_{b} \rho_{b b}\left(v, \omega_{\alpha}, z, t\right)+\gamma_{a b} \rho_{a a}\left(v, \omega_{\alpha}, z, t\right) \\
& -\left[\frac{i \mu}{\hbar} E(z, t) \rho_{b a}\left(v, \omega_{\alpha}, z, t\right)+\text { c.c. }\right] \\
& -\int \Gamma_{b}\left(v^{\prime}, v\right) \rho_{b b}\left(v, \omega_{\alpha}, z, t\right) \mathrm{d} v^{\prime} \\
& +\int \Gamma_{b}\left(v, v^{\prime}\right) \rho_{b b}\left(v^{\prime}, \omega_{\alpha}, z, t\right) \mathrm{d} v^{\prime}, \\
& \rho_{b a}\left(v, \omega_{\alpha}, z, t\right)=\rho_{a b} *\left(v, \omega_{\alpha}, z, t\right),
\end{aligned}
$$

where the subscripts $a$ and $b$ denote the upper and lower laser levels, respectively, $\gamma_{a}$ and $\gamma_{b}$ are the total decay rates for these levels, $\gamma_{a b}$ is the rate of direct decays from level $a$ to level $b, \gamma=\left(\gamma_{a}+\gamma_{b}\right) / 2+\gamma_{p h}$ is the decay rate for the off-diagonal elements with $\gamma_{p h}$ the rate of phase interrupting collisions, $\lambda_{a}$ and $\lambda_{b}$ are the pumping rates, and $\omega_{\alpha}$ is the center frequency of the laser transition for members of an atomic or molecular class $\alpha$. For the case of a single-isotope xenon laser, all the atoms have the same center frequency, so that $\omega_{\alpha}$ would be a constant throughout the calculation. However, it seems likely that similar pulsations will be identified in lasers with other types of inhomogeneous broadening, so the possibility of a distribution of $\omega_{\alpha}$ 's is retained here initially. It should also be noted that at least a few discrete values of $\omega_{\alpha}$ would be required for a realistic description of an ordinary multi-isotope xenon laser.

The meaning of the spectral-cross-relaxation terms of Eqs. (2) and (3) can be best explained by examining a single representative term. Thus the function $\Gamma_{a}\left(v^{\prime}, v\right) \mathrm{d} v^{\prime}$ represents the rate at which atoms in level $a$ having velocities between $v$ and $v+\mathrm{d} v$ will be bumped by means of a collision into a new velocity range between $v^{\prime}$ and $v^{\prime}+\mathrm{d} v^{\prime}$. Conversely, the last integral in Eq. (2) shows atoms in level $a$ being bumped from all possible velocities back into the range between $v$ and $v+$ $\mathrm{d} v$. Similar cross-relaxation integrals have been employed previously in rate-equation treatments of several types of gas lasers, including at least $\mathrm{He}-\mathrm{Ne},{ }^{34,35} \mathrm{CO}_{2},{ }^{36} \mathrm{CO},{ }^{37}$ and xenon $^{38-41}$; and the same ideas have also been employed in semiclassical laser studies. ${ }^{42}$ Spectral cross relaxation can also occur in non-Doppler lasers, and, if necessary, the integrals in Eqs. (2) and (3) could be generalized to include processes that redistribute the intrinsic center frequencies $\omega_{\alpha}$.

To complete the semiclassical model, the Schrödingerequation-based density-matrix equations that have already been written must be joined by a Maxwell-equation-based equation for the electric field. The wave equation for the electric field of a linearly polarized wave in a laser medium can be written as

$$
\frac{\partial^{2} E(z, t)}{\partial z^{2}}-\mu_{0} \sigma \frac{\partial E(z, t)}{\partial t}-\mu_{0} \epsilon_{0} \frac{\partial^{2} E(z, t)}{\partial t^{2}}=\mu_{0} \frac{\partial^{2} P(z, t)}{\partial t^{2}}
$$

The polarization driving this equation can be related back to the off-diagonal density-matrix elements by

$$
P(z, t)=\int_{0}^{\infty} \int_{-\infty}^{\infty} \mu \rho_{a b}\left(v, \omega_{\alpha}, z, t\right) \mathrm{d} v \mathrm{~d} \omega_{\alpha}+\text { c.c. }
$$

Equations (1)-(6) are now a complete set from which the time 
and space dependences of the electric field can be determined, subject to the boundary conditions at the resonator mirrors.

The simplest solutions of Eqs. (1)-(6) are those that apply to one-directional ring lasers, in which both the electric field and the polarization are traveling waves. If the losses in a ring laser can be considered to be uniformly distributed, then the rapid time and space variations in Eqs. (1)-(6) can be factored out by means of the substitutions

$$
\begin{aligned}
E(z, t) & =\frac{1}{2} E^{\prime}(t) \exp (i k z-i \omega t)+\text { c.c. } \\
\rho_{a b}\left(v, \omega_{\alpha}, z, t\right) & =P^{\prime}\left(v, \omega_{\alpha}, t\right) \exp (i k z-i \omega t) / 2 \mu .
\end{aligned}
$$

With the usual rotating-wave approximation these substitutions lead to the new set

$$
\begin{aligned}
\frac{\partial P^{\prime}\left(v, \omega_{\alpha}, t\right)}{\partial t}= & i\left(\omega-\omega_{\alpha}-k v\right) P^{\prime}\left(v, \omega_{\alpha}, t\right)-\gamma P^{\prime}\left(v, \omega_{\alpha}, t\right) \\
& -\frac{i \mu^{2}}{\hbar} E^{\prime}(z, t)\left[\rho_{a a}\left(v, \omega_{\alpha}, t\right)-\rho_{b b}\left(v, \omega_{\alpha}, t\right)\right],
\end{aligned}
$$

$$
\begin{aligned}
\frac{\partial \rho_{a a}\left(v, \omega_{\alpha}, t\right)}{\partial t}= & \lambda_{a}\left(v, \omega_{\alpha}, t\right)-\gamma_{a} \rho_{a a}\left(v, \omega_{\alpha}, t\right) \\
& +\frac{i}{4 \hbar}\left[E^{\prime}(t) P^{\prime *}\left(v, \omega_{\alpha}, t\right)\right. \\
& \left.-E^{\prime *}(t) P^{\prime}\left(v, \omega_{\alpha}, t\right)\right] \\
& -\int \Gamma_{a}\left(v^{\prime}, v\right) \rho_{a a}\left(v, \omega_{\alpha}, t\right) \mathrm{d} v^{\prime} \\
& +\int \Gamma_{a}\left(v, v^{\prime}\right) \rho_{a a}\left(v^{\prime}, \omega_{\alpha}, t\right) \mathrm{d} v^{\prime},
\end{aligned}
$$

$$
\begin{aligned}
\frac{\partial \rho_{b b}\left(v, \omega_{\alpha}, t\right)}{\partial t}= & \lambda_{b}\left(v, \omega_{\alpha}, t\right)-\gamma_{b} \rho_{b b}\left(v, \omega_{\alpha}, t\right) \\
& +\gamma_{a b} \rho_{a a}\left(v, \omega_{\alpha}, t\right)-\frac{i}{4 \hbar}\left[E^{\prime}(t) P^{\prime *}\left(v, \omega_{\alpha}, t\right)\right. \\
& \left.-E^{\prime *}(t) P^{\prime}\left(v, \omega_{\alpha}, t\right)\right] \\
& -\int \Gamma_{b}\left(v^{\prime}, v\right) \rho_{b b}\left(v, \omega_{\alpha}, t\right) \mathrm{d} v^{\prime} \\
& +\int \Gamma_{b}\left(v, v^{\prime}\right) \rho_{b b}\left(v^{\prime}, \omega_{\alpha}, t\right) \mathrm{d} v^{\prime}, \\
\frac{\mathrm{d} E^{\prime}(t)}{\mathrm{d} t}= & -\frac{E^{\prime}(t)}{2 t_{c}}+i(\omega-\Omega) E^{\prime}(t) \\
& +\frac{i \omega_{0} l}{2 \epsilon_{0} L} \int_{0}^{\infty} \int_{-\infty}^{\infty} P^{\prime}\left(v, \omega_{\alpha}, t\right) \mathrm{d} v \mathrm{~d} \omega_{\alpha} .
\end{aligned}
$$

In the field equation, the losses are represented by the cavity lifetime $t_{c}$, higher-order derivatives are neglected, and the results are applied to a laser of background permittivity $\epsilon_{0}$ and optical length $l$ in a cavity of length $L$.

The polarization amplitude $P^{\prime}\left(v, \omega_{\alpha}, t\right)$ and the field amplitude $E^{\prime}(t)$ appearing in Eqs. (9)-(12) are complex quantities. To proceed with the analysis, it is helpful if each of these complex quantities can be expressed in terms of two real quantities. These may be the amplitudes and phases of $P^{\prime}$ and $E^{\prime}$ or the real and imaginary parts of the same parameters. Mixed combinations are possible too, and some authors choose to work with the amplitude and phase of the field together with the real and imaginary parts of the polarization. We find it simplest to use only real and imaginary parts in the forms $P^{\prime}=P_{r}+i P_{i}$ and $E^{\prime}=E_{r}+i E_{i}$. With these substitutions Eqs. (9)-(12) become

$$
\begin{aligned}
\frac{\partial P_{r}}{\partial t}= & -\left(\omega-\omega_{\alpha}-k v\right) P_{i}-\gamma P_{r}+\frac{\mu^{2}}{\hbar} E_{i} D \\
\frac{\partial P_{i}}{\partial t}= & \left(\omega-\omega_{\alpha}-k v\right) P_{r}-\gamma P_{i}-\frac{\mu^{2}}{\hbar} E_{r} D \\
\frac{\partial D}{\partial t}= & \lambda_{a}-\lambda_{b}-\frac{\gamma_{a}+\gamma_{a b}+\gamma_{b}}{2} D \\
& -\frac{\gamma_{a}+\gamma_{a b}-\gamma_{b}}{2} M+\frac{1}{\hbar}\left(E_{r} P_{i}-E_{i} P_{r}\right) \\
& -\frac{1}{2} \int \Gamma_{a}\left(v^{\prime}, v\right)(M+D) \mathrm{d} v^{\prime} \\
& +\frac{1}{2} \int \Gamma_{a}\left(v, v^{\prime}\right)(M+D) \mathrm{d} v^{\prime} \\
& +\frac{1}{2} \int \Gamma_{b}\left(v^{\prime}, v\right)(M-D) \mathrm{d} v^{\prime} \\
& -\frac{1}{2} \int \Gamma_{b}\left(v, v^{\prime}\right)(M-D) \mathrm{d} v^{\prime} \\
\mathrm{d} t & -\frac{E_{i}}{2 t_{c}}+(\omega-\Omega) E_{r}+\frac{\omega_{0} l}{2 \epsilon_{0} L} \int_{0}^{\infty} \int_{-\infty}^{\infty} P_{r} \mathrm{~d} v \mathrm{~d} \omega_{\alpha} \\
& -\frac{E_{r}}{2 t_{c}}-(\omega-\Omega) E_{i}-\frac{\omega_{0} l}{2 \epsilon_{0} L} \int_{0}^{\infty} \int_{-\infty}^{\infty} P_{i} \mathrm{~d} v \mathrm{~d} \omega_{\alpha} \\
& -\frac{1}{2} \int \Gamma_{b}\left(v^{\prime}, v\right)(M-D) \mathrm{d} v^{\prime} \\
& +\frac{1}{2} \int \Gamma_{b}\left(v, v_{a}\left(v_{a}\right)(M-D) \mathrm{d} v^{\prime}\right. \\
& \frac{1}{2} \int \Gamma_{a}\left(v, v^{\prime}\right)(M+D) \mathrm{d} v^{\prime} \\
& =\frac{\gamma_{a}-\gamma_{a b}+\gamma_{b}}{2} M
\end{aligned}
$$

where we have also introduced the population difference $D$ $=\rho_{a a}-\rho_{b b}$ and sum $M=\rho_{a a}+\rho_{b b}$, and, for brevity, the arguments of most of the dependent variables are not explicitly indicated.

Next, it is necessary to make some assumptions about the form of the spectral cross-relaxation kernels. For simplicity we consider the cross-relaxation integrals to apply to "strong" collisions in which the final velocities are distributed randomly across the Doppler profile. Weak velocity-changing collisions primarily broaden the effective homogeneous line shape, and such collisions will be considered below. The strong-collision kernels can thus be written as

$$
\Gamma_{a}\left(v, v^{\prime}\right)=\frac{\Gamma_{a}}{u \pi^{1 / 2}} \exp \left(-v^{2} / u^{2}\right),
$$




$$
\Gamma_{b}\left(v, v^{\prime}\right)=\frac{\Gamma_{b}}{u \pi^{1 / 2}} \exp \left(-v^{2} / u^{2}\right)
$$

where, for example, $\Gamma_{a}$ is the total rate at which atoms in level $a$ undergo strong velocity-changing collisions and $u$ is the most probable speed of the atoms. With these substitutions, Eqs. (15) and (16) for the population difference and sum reduce to

$$
\begin{aligned}
\frac{\partial D}{\partial t}= & \lambda_{a}-\lambda_{b}-\frac{\gamma_{a}{ }^{\prime}+\gamma_{a b}+\gamma_{b}{ }^{\prime}}{2} D \\
& -\frac{\gamma_{a}{ }^{\prime}+\gamma_{a b}-\gamma_{b}{ }^{\prime}}{2} M+\frac{1}{\hbar}\left(E_{r} P_{i}-E_{i} P_{r}\right) \\
& +\frac{\Gamma_{a}}{2 u \pi^{1 / 2}} \exp \left(-v^{2} / u^{2}\right) \int(M+D) \mathrm{d} v^{\prime} \\
& -\frac{\Gamma_{b}}{2 u \pi^{1 / 2}} \exp \left(-v^{2} / u^{2}\right) \int(M-D) \mathrm{d} v^{\prime}
\end{aligned}
$$

$$
\begin{aligned}
\frac{\partial M}{\partial t}= & \lambda_{a}+\lambda_{b}-\frac{\gamma_{a}{ }^{\prime}-\gamma_{a b}-\gamma_{b}{ }^{\prime}}{2} D-\frac{\gamma_{a}^{\prime}-\gamma_{a b}+\gamma_{b}{ }^{\prime}}{2} M \\
& +\frac{\Gamma_{a}}{2 u \pi^{1 / 2}} \exp \left(-v^{2} / u^{2}\right) \int(M+D) \mathrm{d} v^{\prime} \\
& +\frac{\Gamma_{b}}{2 u \pi^{1 / 2}} \exp \left(-v^{2} / u^{2}\right) \int(M-D) \mathrm{d} v^{\prime}
\end{aligned}
$$

where the modified decay rates are defined by $\gamma_{a}{ }^{\prime}=\gamma_{a}+\Gamma_{a}$ and $\gamma_{b}^{\prime}=\gamma_{b}+\Gamma_{b}$. Equations (13), (14), (17), (18), (21), and (22) form the mathematical basis for all the following calculations.

\section{NORMALIZED EQUATIONS}

As a first step in reducing the semiclassical model, it is helpful to rewrite the equations in a normalized form. With an appropriate normalization, these equations are notationally simpler, and they are more clearly expressed in terms of experimentally accessible parameters. A particularly convenient normalization can be obtained from the steady-state version of the governing equations.

The steady-state forms of Eqs. (13), (14), (21), and (22) are

$$
\begin{aligned}
0= & -\left(\omega-\omega_{\alpha}-k v\right) P_{i}-\gamma P_{r}+\frac{\mu^{2}}{\hbar} E_{i} D, \\
0= & \left(\omega-\omega_{\alpha}-k v\right) P_{r}-\gamma P_{i}-\frac{\mu^{2}}{\hbar} E_{r} D, \\
0= & \lambda_{a}-\lambda_{b}-\frac{\gamma_{a}^{\prime}+\gamma_{a b}+\gamma_{b}^{\prime}}{2} D \\
& -\frac{\gamma_{a}^{\prime}+\gamma_{a b}-\gamma_{b}^{\prime}}{2} M+\frac{1}{\hbar}\left(E_{r} P_{i}-E_{i} P_{r}\right) \\
& +\frac{\Gamma_{a}}{2 u \pi^{1 / 2}} \exp \left(-v^{2} / u^{2}\right) \int(M+D) \mathrm{d} v^{\prime} \\
& -\frac{\Gamma_{b}}{2 u \pi^{1 / 2}} \exp \left(-v^{2} / u^{2}\right) \int(M-D) \mathrm{d} v^{\prime},
\end{aligned}
$$

$$
\begin{aligned}
0= & \lambda_{a}+\lambda_{b}-\frac{\gamma_{a}{ }^{\prime}-\gamma_{a b}-\gamma_{b}{ }^{\prime}}{2} D-\frac{\gamma_{a}{ }^{\prime}-\gamma_{a b}+\gamma_{b}{ }^{\prime}}{2} M \\
& +\frac{\Gamma_{a}}{2 u \pi^{1 / 2}} \exp \left(-v^{2} / u^{2}\right) \int(M+D) \mathrm{d} v^{\prime} \\
& +\frac{\Gamma_{b}}{2 u \pi^{1 / 2}} \exp \left(-v^{2} / u^{2}\right) \int(M-D) \mathrm{d} v^{\prime}
\end{aligned}
$$

Equations (23) and (24) can be solved for the polarizations $P_{r}$ and $P_{i}$, and the results are

$$
\begin{aligned}
& P_{r}=\frac{\mu^{2} D}{\gamma \hbar} \frac{\left[\left(\omega-\omega_{\alpha}-k v\right) / \gamma\right] E_{r}+E_{i}}{1+\left[\left(\omega-\omega_{\alpha}-k v\right) / \gamma\right]^{2}}, \\
& P_{i}=\frac{\mu^{2} D}{\gamma \hbar} \frac{\left[\left(\omega-\omega_{\alpha}-k v\right) / \gamma\right] E_{i}-E_{r}}{1+\left[\left(\omega-\omega_{\alpha}-k v\right) / \gamma\right]^{2}} .
\end{aligned}
$$

With these substitutions, Eqs. (25) and (26) become

$$
\begin{aligned}
0= & \lambda_{a}-\lambda_{b}-\frac{\gamma_{a}{ }^{\prime}+\gamma_{a b}+\gamma_{b}^{\prime}}{2} D \\
& -\frac{\gamma_{a}{ }^{\prime}+\gamma_{a b}-\gamma_{b}{ }^{\prime}}{2} M-\frac{\mu^{2} D}{\gamma \hbar^{2}} \frac{E_{r}{ }^{2}+E_{i}^{2}}{1+\left[\left(\omega-\omega_{\alpha}-k v\right) / \gamma\right]^{2}} \\
& +\frac{\Gamma_{a}}{2 u \pi^{1 / 2}} \exp \left(-v^{2} / u^{2}\right) \int(M+D) \mathrm{d} v^{\prime} \\
& -\frac{\Gamma_{b}}{2 u \pi^{1 / 2}} \exp \left(-v^{2} / u^{2}\right) \int(M-D) \mathrm{d} v^{\prime} \\
& =\lambda_{a}+\lambda_{b}-\frac{\gamma_{a}^{\prime}-\gamma_{a b}-\gamma_{b}^{\prime}}{2} D \\
& -\frac{\gamma_{a}{ }^{\prime}-\gamma_{a b}+\gamma_{b}^{\prime}}{2} M \\
& +\frac{\Gamma_{a}}{2 u \pi^{1 / 2}} \exp \left(-v^{2} / u^{2}\right) \int(M+D) \mathrm{d} v^{\prime} \\
& +\frac{\Gamma_{b}}{2 u \pi^{1 / 2}} \exp \left(-v^{2} / u^{2}\right) \int(M-D) \mathrm{d} v^{\prime}
\end{aligned}
$$

The next step in the analysis is to obtain formal solutions for the population parameters $D$ and $M$. For compactness it is helpful to define the new pump rates

$$
\begin{aligned}
& \lambda_{a}{ }^{\prime}=\left[L_{a}+\frac{\Gamma_{a}}{2} \int(M+D) \mathrm{d} v^{\prime}\right] \frac{\exp \left(-v^{2} / u^{2}\right)}{u \pi^{1 / 2}}, \\
& \lambda_{b}{ }^{\prime}=\left[L_{b}+\frac{\Gamma_{b}}{2} \int(M-D) \mathrm{d} v^{\prime}\right] \frac{\exp \left(-v^{2} / u^{2}\right)}{u \pi^{1 / 2}},
\end{aligned}
$$

where the Gaussian velocity distribution in the pump functions has been factored out according to

$$
\begin{aligned}
& \lambda_{a}=L_{a} \exp \left(-v^{2} / u^{2}\right) / u \pi^{1 / 2}, \\
& \lambda_{b}=L_{b} \exp \left(-v^{2} / u^{2}\right) / u \pi^{1 / 2} .
\end{aligned}
$$

With this notation Eqs. (29) and (30) simplify to

$$
\begin{aligned}
0= & \lambda_{a}{ }^{\prime}-\lambda_{b}{ }^{\prime}-\frac{\gamma_{a}{ }^{\prime}+\gamma_{a b}+\gamma_{b}{ }^{\prime}}{2} D-\frac{\gamma_{a}{ }^{\prime}+\gamma_{a b}-\gamma_{b^{\prime}}}{2} M \\
& -\frac{\mu^{2} D}{\gamma \hbar^{2}} \frac{E_{r}{ }^{2}+E_{i}{ }^{2}}{1+\left[\left(\omega-\omega_{\alpha}-k v\right) / \gamma\right]^{2}}, \\
0= & \lambda_{a}{ }^{\prime}+\lambda_{b}{ }^{\prime}-\frac{\gamma_{a}{ }^{\prime}-\gamma_{a b}-\gamma_{b}{ }^{\prime}}{2} D-\frac{\gamma_{a}{ }^{\prime}-\gamma_{a b}+\gamma_{b}{ }^{\prime}}{2} M
\end{aligned}
$$


If $M$ is eliminated between these equations, one finds that the population difference can be written as

$$
D=\frac{\left(1-\gamma_{a b} / \gamma_{b}{ }^{\prime}\right)\left(\lambda_{a}{ }^{\prime} / \gamma_{a}{ }^{\prime}\right)-\lambda_{b}{ }^{\prime} / \gamma_{b}{ }^{\prime}}{1+\left(A_{r}{ }^{2}+A_{i}{ }^{2}\right) /\left[1+\left(\omega-\omega_{\alpha}-k v\right)^{2} / \gamma^{2}\right]},
$$

where we have introduced the normalized real and imaginary field components

$$
\begin{aligned}
& A_{r}=\left(\frac{\gamma_{a}{ }^{\prime}-\gamma_{a b}+\gamma_{b}}{2 \gamma \gamma_{a}^{\prime} \gamma_{b^{\prime}}}\right)^{1 / 2} \frac{\mu E_{r}}{\hbar}, \\
& A_{i}=\left(\frac{\gamma_{a}^{\prime}-\gamma_{a b}+\gamma_{b}{ }^{\prime}}{2 \gamma \gamma_{a}^{\prime} \gamma_{b^{\prime}}}\right)^{1 / 2} \frac{\mu E_{i}}{\hbar} .
\end{aligned}
$$

Alternatively, one could express the saturation denominator in Eq. (37) in terms of the field intensity $A A^{*}=A_{r}{ }^{2}+{A_{i}}^{2}$.

It is helpful now to write the dynamic equations of the laser in terms of the normalized field amplitudes that have been defined in Eqs. (38) and (39). Doing this also leads to the useful new normalization for the polarization, population, and pump terms

$$
\begin{aligned}
& P_{i}(V, U)=\epsilon u \gamma \frac{t_{c} \omega_{0} l}{\epsilon_{0} L} \frac{\mu}{\hbar}\left(\frac{\gamma_{a}^{\prime}-\gamma_{a b}+\gamma_{b}^{\prime}}{2 \gamma \gamma_{a}^{\prime} \gamma_{b}^{\prime}}\right)^{1 / 2} P_{i}\left(v, \omega_{\alpha}\right) \\
& P_{r}(V, U)=\epsilon u \gamma \frac{t_{c} \omega_{0} l}{\epsilon_{0} L} \frac{\mu}{\hbar}\left(\frac{\gamma_{a^{\prime}-\gamma_{a b}+\gamma_{b}^{\prime}}}{2 \gamma \gamma_{a}^{\prime} \gamma_{b^{\prime}}}\right)^{1 / 2} P_{r}\left(v, \omega_{\alpha}\right) \\
& D(V, U)=\frac{\epsilon u t_{c} \omega_{0} l \mu^{2}}{\epsilon_{0} L \hbar} D\left(v, \omega_{\alpha}\right) \\
& M(V, U)=\frac{\epsilon u t_{c} \omega_{0} l \mu^{2}}{\epsilon_{0} L \hbar} M\left(v, \omega_{\alpha}\right) \\
& \lambda_{a}(V, U)=\frac{\epsilon u t_{c} \omega_{0} l \mu^{2}}{\epsilon_{0} L \hbar} \lambda_{a}\left(v, \omega_{\alpha}\right) \\
& \lambda_{b}(V, U)=\frac{\epsilon u t_{c} \omega_{0} l \mu^{2}}{\epsilon_{0} L \hbar} \lambda_{b}\left(v, \omega_{\alpha}\right)
\end{aligned}
$$

where we have introduced the new variables

$$
\begin{aligned}
& V=\frac{v}{\epsilon u}=\frac{k v}{\gamma}, \\
& U=\frac{\omega_{\alpha}-\omega_{0}}{\gamma},
\end{aligned}
$$

and $\epsilon=\left(\Delta \nu_{h} / \Delta \nu_{d}\right)(\ln 2)^{1 / 2}$ is the natural damping ratio. ${ }^{43}$ With these substitutions, Eqs. (13), (14), (17), (18), (21), and (22) take on the relatively simple forms

$$
\begin{aligned}
& \frac{\partial P_{r}}{\partial t}=-\gamma\left[P_{r}+(y-U-V) P_{i}-A_{i} D\right], \\
& \frac{\partial P_{i}}{\partial t}=-\gamma\left[P_{i}-(y-U-V) P_{r}+A_{r} D\right],
\end{aligned}
$$

$$
\begin{aligned}
\frac{\partial D}{\partial t}= & \lambda_{a}-\lambda_{b}-\frac{\gamma_{a}{ }^{\prime}+\gamma_{a b}+\gamma_{b}^{\prime}}{2} D \\
& -\frac{\gamma_{a}^{\prime}+\gamma_{a b}-\gamma_{b}^{\prime}}{2} M \\
& +\frac{2 \gamma_{a}^{\prime} \gamma_{b}^{\prime}}{\gamma_{a}^{\prime}-\gamma_{a b}+\gamma_{b}^{\prime}}\left(A_{r} P_{i}-A_{i} P_{r}\right) \\
& +\frac{\epsilon \Gamma_{a}}{2 \pi^{1 / 2}} \exp \left(-\epsilon^{2} V^{2}\right) \int_{-\infty}^{\infty}(M+D) \mathrm{d} V^{\prime} \\
& -\frac{\epsilon \Gamma_{b}}{2 \pi^{1 / 2}} \exp \left(-\epsilon^{2} V^{2}\right) \int_{-\infty}^{\infty}(M-D) \mathrm{d} V^{\prime}, \\
\frac{\partial M}{\partial t}= & \lambda_{a}+\lambda_{b}-\frac{\gamma_{a}^{\prime}-\gamma_{a b}-\gamma_{b}^{\prime}}{2} D-\frac{\gamma_{a}^{\prime}-\gamma_{a b}+\gamma_{b}^{\prime}}{2} M \\
& +\frac{\epsilon \Gamma_{a}}{2 \pi^{1 / 2}} \exp \left(-\epsilon^{2} V^{2}\right) \int_{-\infty}^{\infty}(M+D) \mathrm{d} V^{\prime} \\
& +\frac{\epsilon \Gamma_{b}}{2 \pi^{1 / 2}} \exp \left(-\epsilon^{2} V^{2}\right) \int_{-\infty}^{\infty}(M-D) \mathrm{d} V^{\prime}, \\
\frac{\partial A_{r}}{\partial t}= & -\frac{1}{2 t_{c}}\left[A_{r}+\delta\left(y-y_{0}\right) A_{i}+\int_{-\infty}^{\infty} \int_{-\infty}^{\infty} P_{i} \mathrm{~d} V \mathrm{~d} U\right], \\
\frac{\partial A_{i}}{\partial t}= & -\frac{1}{2 t_{c}}\left[A_{i}-\delta\left(y-y_{0}\right) A_{r}-\int_{-\infty}^{\infty} \int_{-\infty}^{\infty} P_{r} \mathrm{~d} V \mathrm{~d} \dot{U}\right],
\end{aligned}
$$

where we have also introduced the normalized lasing frequency $y=\left(\omega-\omega_{0}\right) / \gamma$, the normalized cavity frequency $y_{0}$ $=\left(\Omega-\omega_{0}\right) / \gamma$, and the decay parameter $\delta=2 \gamma t_{c}$.

Equations (48)-(53) allow for the possibility of a distribution of the natural transition center frequencies ( $U$ in the normalized units). However, available pulsation data for xenon lasers have mostly been obtained using monoisotopic xenon. Consequently, the dependent variables in these equations may, for present purposes, be regarded as delta functions of $U$. With this understanding, the form of Eqs. (48)-(53) remains unchanged, provided that $U$ is set equal to zero in Eqs. (48) and (49) and the integral over $U$ is removed from Eqs. (52) and (53). This reduced form of the equations is the basis for the numerical solutions described in Section 5.

\section{DATA}

In the previous discussions, a theoretical model has been developed for interpreting and predicting the spontaneouspulsation characteristics of practical laser oscillators. In order to apply these results to any specific laser, it is necessary to obtain values for the numerous parameters that appear in the model. As mentioned above, our emphasis here is on the xenon laser operating at $3.51-\mu \mathrm{m}$ wavelength. Thus the purpose of this section is to extract from published results accurate values for the parameters of a xenon laser. The following section describes various predictions of the model when these parameter values are employed.

Among the most fundamental parameters of a xenon laser are the lifetimes of the upper and lower laser levels. The upper level corresponding to the 3.51- $\mu \mathrm{m}$ transition is identified as $5 d[7 / 2]_{3}^{\circ}$, and the lower level is $6 p[5 / 2]_{2}$. The lifetimes 
Table 1. Xenon-Laser Lifetimes

\begin{tabular}{|c|c|c|c|}
\hline & $\tau_{a}$ (nsec) & $\tau_{b}$ (nsec) & $\Delta \nu_{n}(\mathrm{MHz})$ \\
\hline Clark et al..$^{44}$ & 1350 & 44 & 3.74 \\
\hline Allen et al..$^{45}$ & 1365 & 45 & 3.65 \\
\hline $\begin{array}{l}\text { Husson and Margerie }{ }^{46} \\
\text { and Aymar }\end{array}$ & $1200^{a}$ & $35^{a}$ & 4.68 \\
\hline
\end{tabular}

${ }^{a}$ Ref. 48.

of these levels have been computed many times, and the results of some typical calculations are included in Table 1. Also shown in the table is the Lorentz (natural) linewidth implied by these lifetimes using the formula $\Delta \nu_{n}=\left(\tau_{a}{ }^{-1}+\right.$ $\left.\tau_{b}{ }^{-1}\right) / 2 \pi$. It is difficult to obtain a direct measurement of these decay times, but there are various techniques that could in principle be used to determine the homogeneous linewidth. Unfortunately, at normal operating pressures, the homogeneous linewidth is dominated by Holtsmark (pressure) broadening, and the Lorentz linewidth must be determined by extrapolating experimental data to zero pressure. Studies of this type yield good agreement with the linewidth values shown in Table 1, but they may not have sufficient resolution to select a particular set of lifetimes from this table. Nevertheless, to be specific and consistent with several recent investigations, we have adopted the values of Husson and Margerie ${ }^{46}$ and Aymar, ${ }^{47}$ namely, $\tau_{a}=1200$ nsec and $\tau_{b}=35$ nsec. Another important parameter in these studies is the upper-state branching ratio. In particular, it is necessary to obtain a value for $\gamma_{a b}$. It has been shown experimentally 49 and theoretically ${ }^{50}$ that almost every spontaneous decay from the upper laser level of xenon leads directly to the lower level. Consequently, for our xenon calculations we have used the relationship $\gamma_{a b} \simeq \gamma_{a}$.

The values of the pressure-broadening coefficients for the xenon laser at $3.51-\mu \mathrm{m}$ wavelength have also been reported, but the actual numerical values quoted depend somewhat on the theoretical procedure chosen to interpret the experimental data. Based on saturated spectroscopy measurements, Nella et al. have reported a value for the phase-interrupting collision rate of $\gamma_{p h}=\left(4.5 \times 10^{-9}\right)^{-1} P \mathrm{sec}^{-1}$, where $P$ is the pressure in Torr. ${ }^{51}$ Cahuzac et al. obtained similar but more detailed results and found that the effective homogeneous-broadening coefficient is a nonlinear function of pressure. ${ }^{39}$ In linearabsorption laser experiments it has been shown that for most pressures of interest the true phase-interruption rate can be written $\gamma_{p h}=\pi \times 10.9 \times 10^{6} P \mathrm{sec}^{-1}, 48$ while the additional nonlinear terms are a consequence of velocity-changing collisions. ${ }^{40,48}$ In our calculations, we have adopted this value for $\gamma_{p h}$. Our experimental lasers have generally operated at the vapor pressure of xenon at $77 \mathrm{~K}$ or 5 mTorr (Ref. 52) [or $3 \mathrm{~m}$ Torr (Ref. 39)], and thus the phase-decay rate assumed here is $\gamma_{p h}=0.17 \times 10^{6} \mathrm{sec}^{-1}$.

As just mentioned, the actual homogeneous linewidth includes important contributions due to velocity-changing collisions, and for our theoretical model these contributions must be included in the calculations of the coefficient $\gamma$. It follows from the data of Cahuzac et al. that, at our operating pressure, the effective homogeneous linewidth is about 5.2 MHz. ${ }^{39}$ Since, as noted in Table 1 , the natural linewidth is about $4.7 \mathrm{MHz}$ and phase-interrupting collisions are relatively unimportant, it follows that velocity-changing collisions are contributing about $0.5 \mathrm{MHz}$ to the effective homogeneous linewidth. The coefficient $\gamma$ for use in our calculations is $\pi$ $\times 5.2 \times 10^{6}=16.3 \times 10^{6} \mathrm{sec}^{-1}$.

A value is also required for the rate of strong velocitychanging collisions. Cahuzac et al. have deduced that the rate of weak velocity-changing collisions in xenon is roughly 2.5 $\times 10^{8} \mathrm{sec}^{-1}$ Torr $^{-1}$, which in our studies would yield a rate of about $1.25 \times 10^{6} \mathrm{sec}^{-1.40}$ With perturbing atoms having a mass as large as or larger than the mass of the lasing atoms, a substantial percentage of all velocity-changing collisions may be regarded as strong collisions that cause the atoms to nearly forget their initial velocities. ${ }^{41}$ As a result, we may assume that the rate $\Gamma_{a}$ of strong velocity-changing collisions affecting atoms in the upper laser level will be on the order of $1 \times 10^{6}$ $\mathrm{sec}^{-1}$, and a similar value should apply to the lower laser level. We regard this estimate as quite uncertain, and, in calculations similar to those described in Section 5 , it has been confirmed that substantially larger or smaller values of the strong velocity-changing collision rates result in poorer agreement with experiment.

The Doppler width for the $3.51-\mu \mathrm{m}$ transition in xenon is reasonably well known. Under low current conditions of 1.6 $\mathrm{mA} / \mathrm{cm}^{2}$, the gas remains close to room temperature and the Doppler width is about $91 \mathrm{MHz} .{ }^{48}$ Under lasing conditions, the temperature may be close to $500 \mathrm{~K}\left(\Delta \nu_{d} \simeq 120 \mathrm{MHz}\right),{ }^{53}$ and to be specific we have continued to use the intermediate value of $\Delta \nu_{d}=110 \mathrm{MHz}{ }^{11}$ Thus the natural damping ratio used in our calculations is $\epsilon=\Delta \nu_{h}(\ln 2)^{1 / 2} / \Delta_{d}=$ $5.2(\ln 2)^{1 / 2} / 110=0.039$.

Finally, it is important to have some estimate of the relative size of the pumping rates into the upper and lower states of the laser transition. According to Klüver, the unsaturated population ratio in a xenon-laser amplifer is about $n_{a} / n_{b} \simeq$ 2.6. ${ }^{54}$ Degeneracy factors are not important when relating populations to the unsaturated gain, and spectral cross-relaxation effects also cancel out.55 Recalling the approximate equality $\gamma_{a b} \simeq \gamma_{a}$, the ratio of pumping rates can be shown to be

$$
\frac{\lambda_{b}}{\lambda_{a}}=\frac{\gamma_{b} n_{b}}{\gamma_{a} n_{a}}-1 .
$$

Using the values obtained above, this is $\lambda_{b} / \lambda_{a}=12.2$.

\section{NUMERICAL SOLUTIONS}

There are several basic features of the experimental spontaneous-pulsation data that a meaningful theoretical model must be able to reproduce. Some of these features are illustrated in Fig. 1. First and most obviously, the model must be capable of predicting low-threshold spontaneous pulsations. The individual pulses may be quite complicated in shape, and the overall pulse train may not even be periodic. As will be discussed below, the model that has been developed in the previous sections predicts all these features and in fact seems to provide quantitative agreement with available experimental data. It should be admitted, however, that the experimental data included here were obtained using a standing-wave xenon laser detuned from line center. Although a detuned standing-wave laser can, in principle, act like a traveling-wave ring laser, further studies of spontaneous pulsations in standingwave lasers are needed and are in progress. 

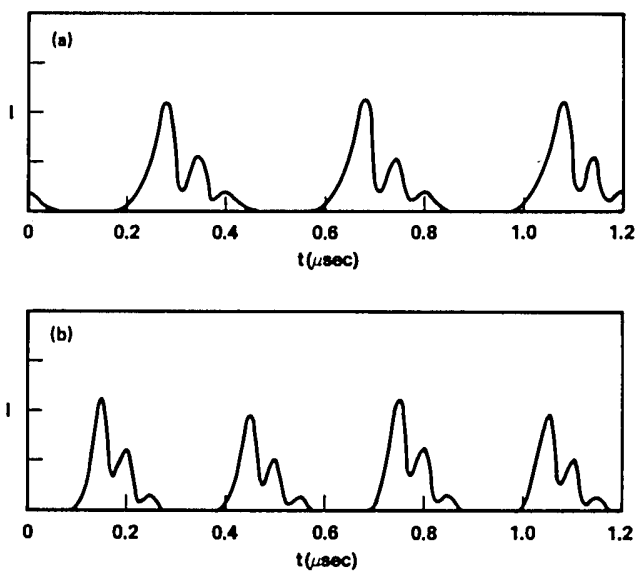

Fig. 1. Experimental pulsation waveforms for (a) a discharge current of $40 \mathrm{~mA}$ and (b) a discharge current of $50 \mathrm{~mA}$ in a xenon laser. These curves are reproduced from Ref. 11, and further experimental details are in that reference.

The starting point for our numerical calculations is the equation set Eqs. (48)-(53). The procedures employed here are similar to those used in our previous study of Lambequation instability. ${ }^{11}$ In particular, the time integrations have been performed using a modified second-order RungeKutta method, and after each time step the velocity integrals have been recalculated using Simpson's rule. Other techniques have also been introduced for relating the pump rates to the threshold parameter $r$, for minimizing the computer run time, etc. In this study the threshold parameter is defined as the ratio of the unsaturated line-center gain to the gain value required to reach threshold for line-center tuning.

The solutions of the laser equations present a wealth of curious transient phenomena, and it is helpful first simply to display a series of plots showing the time development of the laser intensity. Such a series is shown in Fig. 2 with linecenter tuning and decreasing values of the threshold parameter $r$. All the physical laser parameters used in obtaining these plots are as deduced in Section 4, except for the cavity lifetime $t_{c}$. The cavity lifetime has been varied a bit, and we find that a lifetime of $1.0 \mathrm{nsec}$, as used previously, ${ }^{11}$ leads to better agreement with experimental data than values a factor of 2 larger or smaller. Hence we have continued to use $t_{c}=$ $1.0 \mathrm{nsec}$.

The plot of normalized intensity $I$ versus time that is shown in Fig. 2(a) corresponds to line-center operation of the xenon laser far above threshold with a threshold parameter of $r=$ 3.0. This single plot illustrates many features of the wonderfully complex xenon-laser behavior. First, and most obviously, the laser is unstable under these conditions. The output is deeply modulated and often appears as semiperiodic bursts of short pulses. The principal period of these bursts is about $0.2 \mu \mathrm{sec}$. However, if one observes that the bursts are approximately alternating in form, it could be argued that the period is better described as $0.4 \mu \mathrm{sec}$. But in fact the pulses in this example seem to be fundamentally chaotic with no exact long-term periodicity. With line-center tuning the intensity goes to zero between subpulses, but this circumstance is slightly obscured by the limited resolution of the plot.

The next plot, shown in Fig. 2(b), corresponds to a smaller threshold parameter of $r=2.5$. In this case, the pulsation frequency is lower, and the intensity repeats exactly on alternate bursts. Even though the output is quite complex, it is not chaotic in the conventional sense.

For slightly lower values of the threshold parameter the laser enters a region of very complex behavior in which each
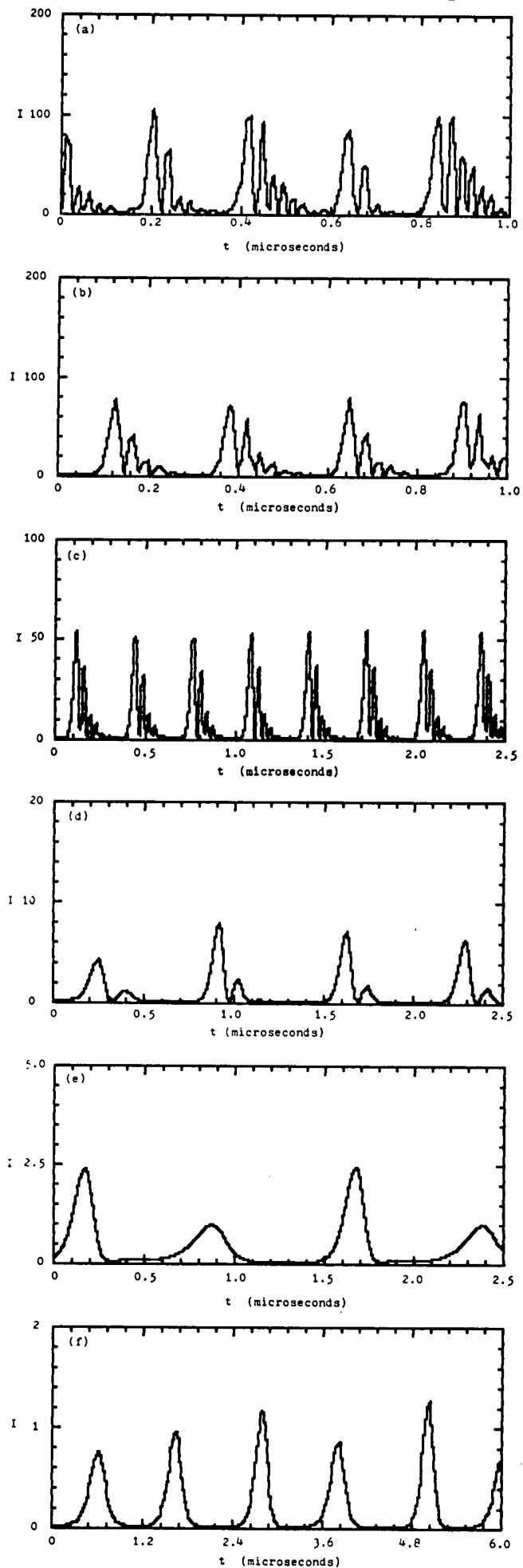

Fig. 2. Theoretical spontaneous-pulsation waveforms for a singlemode xenon laser with line-center tuning and the threshold-parameter values: (a) $r=3.0$, (b) $r=2.5$, (c) $r=2.0$, (d) $r=1.2$, (e) $r=1.1$, and (f) $r=1.06$. Generally, the pulsation amplitude, frequency, and waveform complexity decrease with decreasing values of $r$. Transitions between periodic waveform types are usually chaotic. 
i (ma)

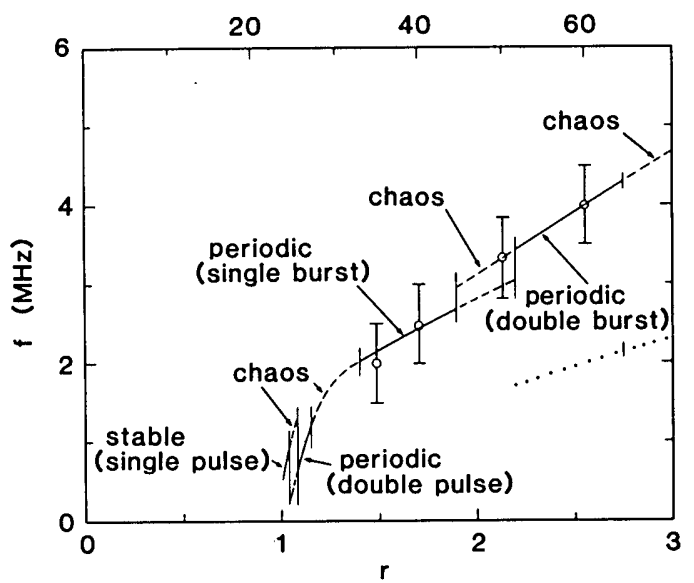

Fig. 3. Theoretical (lines) and experimental (circles) pulsation frequencies as a function of threshold parameter (discharge current). Dashed lines indicate the regions of chaotic output, and the dotted line is meant to emphasize the fact that in the double-burst region the true repetition frequency is half of what is indicated by the solid line.

intensity burst is more similar to its immediate predecessor with exact or almost exact burst repetition occurring after increasing numbers of bursts. In the limit of this process, successive bursts are exactly alike, and this limit has almost been reached for an $r$ value of 2.0, as shown in Fig. 2(c). In this sequence, the drift from burst to burst is very slight and the long-range repetition period is already several microseconds.

This behavior of exactly periodic pulsation bursts continues for threshold parameters down to about $r=1.4$. At this point, another region of chaos is entered, indicative of a transition to a fundamentally different type of pulsation waveform. An example of this chaotic behavior is given in Fig. 2(d) for an $r$ value of 1.2. The pulses here have a lower repetition rate, and, except for the first echo pulse, the secondary pulses have damped away.

The next step in this process is illustrated in Fig. 2(e) for an $r$ value of 1.1. Here, the echo pulse has drifted back until it occurs about halfway between the principal pulses, and the pulse train is again exactly periodic. With further decreases in $r$, yet another region of chaos is encountered as the height of the midpulse increases to equality with the principal pulse. An example of such a sequence is shown in Fig. 2(f) for a threshold parameter of $r=1.06$. With $r$ near 1.05, the pulse train is exactly periodic, having equal-amplitude pulses. For still smaller values of $r$ the laser is stable, so that for any initial conditions the pulsations damp toward a steady-state output.

Figure 3 summarizes much of the information that has just been discussed. This figure includes a theoretical plot of the spontaneous-pulsation frequency as a function of the threshold parameter $r$ for a ring laser tuned to line center, and these quantitative results are reminiscent of the simple analytical frequency curves discussed previously. ${ }^{17,18}$ This plot shows the regions of chaos that separate the regions of regular pulsations. It should be noted that even within the chaos regions there may be narrow areas of long period repetition. For reasons of clarity, no attempt is made to represent these areas on the plot. Also shown in the figure are some of the experimental pulsation-frequency data that the theoretical curve is intended to represent. ${ }^{11}$ The gain of low-pressure xenon lasers is found to vary linearly with discharge current for this region of operation, ${ }^{9}$ and this fact together with threshold current measurements is used to establish the scale for plotting the data points. The single-burst and doubleburst pulsation regions and waveforms are in excellent agreement with experimental data such as those shown in Fig. 1.

One result of these studies is that changes from one type of periodicity to another are generally accompanied by a region of chaotic pulsations. Also, there are, typically, substantial changes in the pulsation frequency and the rate of change of frequency with pumping (slope in the plot) as the laser crosses a region of chaos. Thus the chaos can be understood as resulting from the nonlinear interaction between the tendency to pulse with the frequency and waveform extrapolated from above the chaos region and the tendency to pulse with the incommensurate frequency and waveform extrapolated from below the chaos region.

Besides the pulsation wave shape and frequency, it is also of interest to consider the time-averaged laser intensity. In Fig. 4 are plots of the average laser intensity as a function of the threshold parameter $r$. The upper plot has been obtained by numerically averaging the laser pulsation waveforms. The lower plot shows the steady-state intensity solutions of the same equations. The most striking feature of these results is the dramatic increase in average power that occurs when the laser is pulsing. This increase in average power associated with a pulsing output has long been known in other studies of optical oscillations and has been attributed to an underlying "maximum emission principle." 56 Although this principle is not always consistent with the more fundamental Hamilton's principle, it may provide a useful context for interpreting existing instability data. ${ }^{57}$

The regions of chaotic output are also indicated in Fig. 4. It is interesting to note that with increased pumping the transitions to more complex pulsation waveforms may be accompanied by an increase in slope of the intensity-versusthreshold-parameter curve. Of the many solution types that

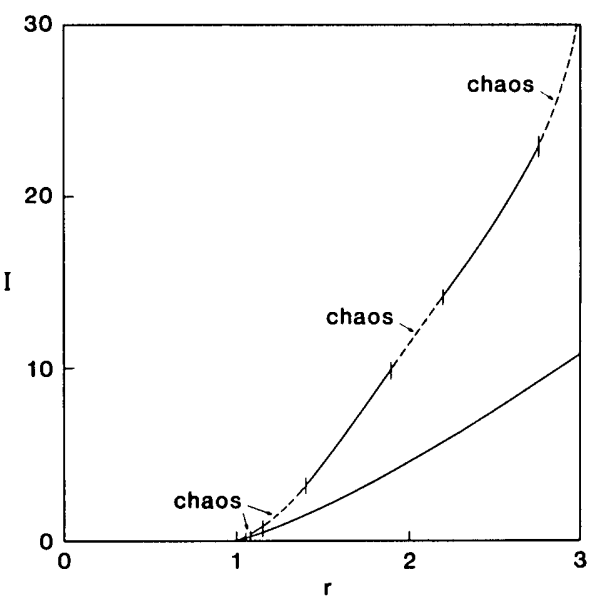

Fig. 4. Theoretical normalized-average output intensity as a function of threshold parameter for a laser tuned to line center. Dashed lines indicate the regions of chaos, and the lower curve shows the steadystate intensity that would result if all time derivatives were set to zero. 

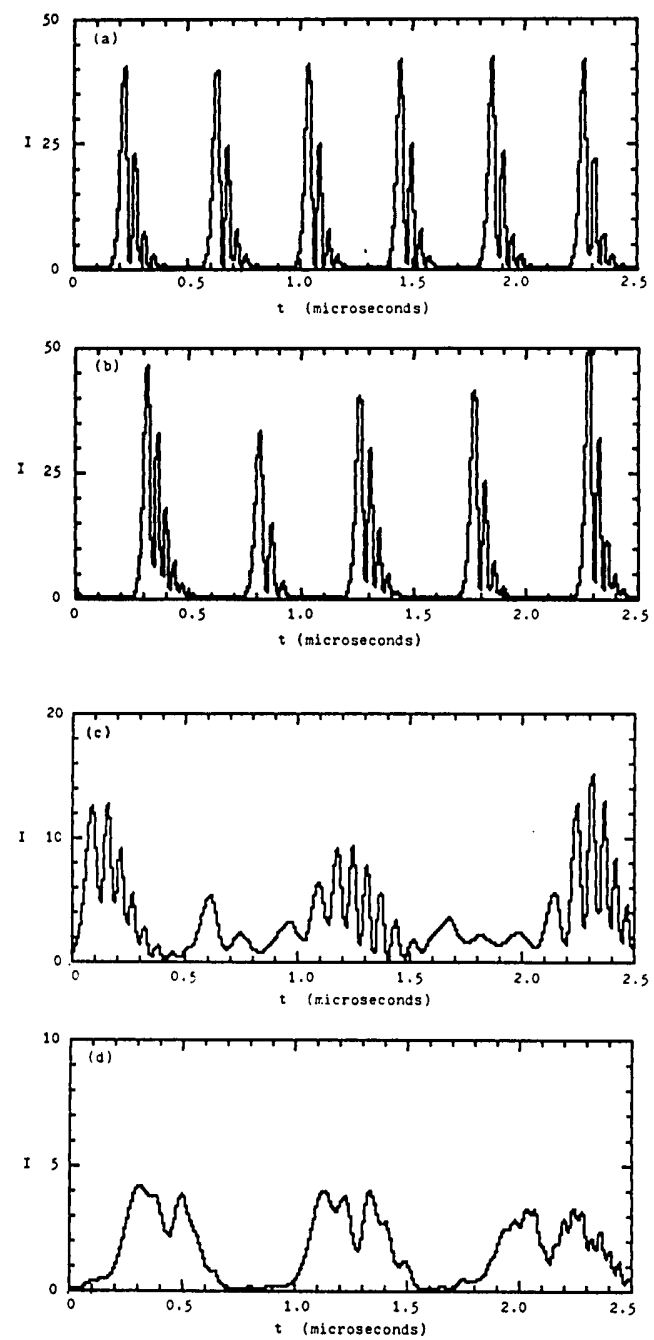

Fig. 5. Theoretical spontaneous-pulsation waveforms for a singlemode xenon laser with a threshold parameter of $r=1.7$ and detunings of (a) $\Delta \nu=0$, (b) $\Delta \nu=2.5 \Delta \nu_{h}$, (c) $\Delta \nu=5 \Delta \nu_{h}$, and (d) $\Delta \nu=$ $7.5 \Delta \nu_{h}$.

are possible well above threshold, the one that yields the largest average intensity may be the one that is selected. The pràctical consequence of these results is that neglecting the possibility of pulsations in a theoretical study may lead to a substantial underestimate of the average laser output power. For nonlinear applications it is especially important to recognize that under pulsating conditions this enhanced average power is condensed into rather sharp spikes, which would further enhance the beam effectiveness.

The model that has been developed here can also be applied to study the effects of cavity detuning on the pulsation characteristics. A series of pulsation waveforms for various values of detuning is shown in Fig. 5. All these pulsations are obtained with a threshold parameter of $r=1.7$. With zero detuning, the pulsations are essentially periodic with the output appearing as a train of nearly identical bursts. Detuning the laser from line center generally tends to increase the complexity of the output. With a detuning of 2.5 homogeneous linewidths, the output bursts have ceased to be identical, and the intensity no longer vanishes between the smaller secondary pulses. With a detuning of $5.0 \Delta \nu_{h}$, the pulses are more irregular, and the intensity remains substantial between the major bursts. Still stranger pulses are observed with a detuning of $7.5 \Delta \nu_{h}$.

The laser model can also be readily applied to lasers under other operating conditions. The pulsation waveforms ob-
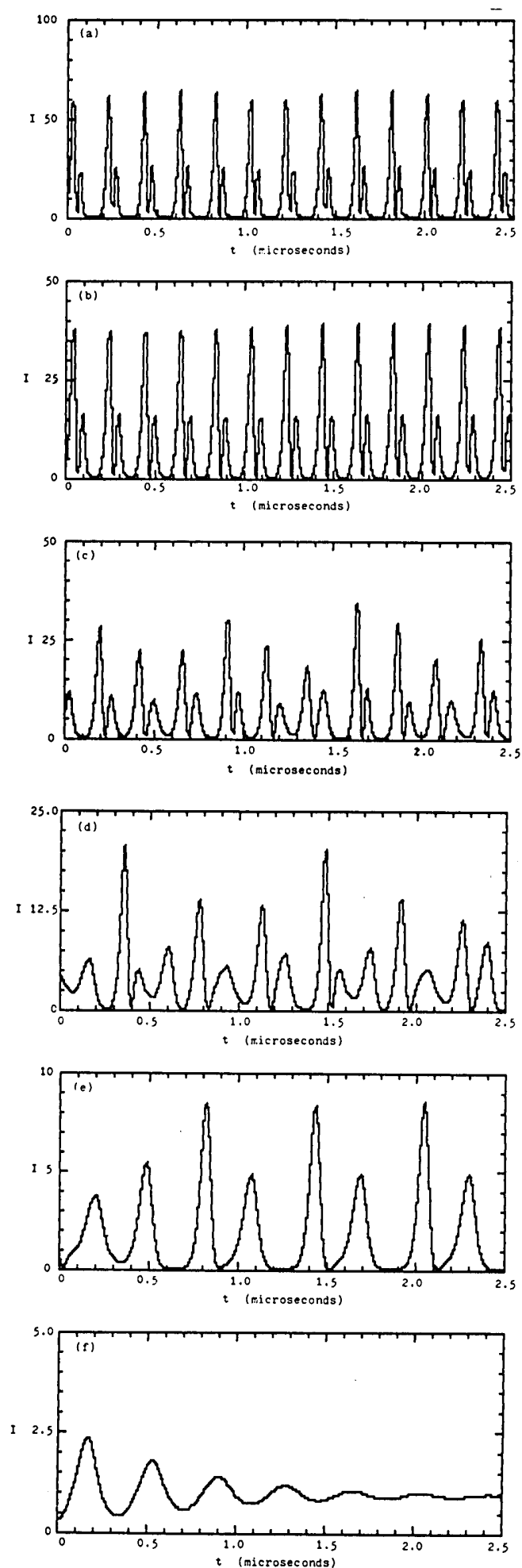

Fig. 6. Theoretical spontaneous-pulsation waveforms for a higherpressure (50-mTorr) single-mode xenon laser with line-center tuning and the threshold-parameter values (a) $r=2.0$, (b) $r=1.8,(c) r=1.6$, (d) $r=1.4$, (e) $r=1.2$, and (f) $r=1.1$. Pulsations are faster, exhibit less ringing, and have a higher instability threshold than the lowerpressure situation represented in Fig. 2 . 
tained by Maeda and Abraham ${ }^{24}$ and by Abraham et al. ${ }^{12}$ generally correspond to much higher xenon pressure than used in our own experiments. To indicate the consequences of higher pressure and to represent these additional data better, we have adjusted the relaxation rates in the model to correspond to a xenon pressure of $50 \mathrm{~m}$ Torr rather than $5 \mathrm{mTorr}$. In principle, the line-broadening effects of helium buffer gas could also be included since the appropriate coefficients have been reported. ${ }^{48}$ However, our only purpose here is to indicate the qualitative effects of higher pressure, and, for simplicity, helium is assumed to be absent. The results are shown in Fig. 6 for line-center tuning and a range of threshold-parameter values. A comparison of Figs. 2 and 6 shows that for similar values of the threshold parameter the higher-pressure laser pulses faster with simpler pulsation waveforms. Also, the threshold for pulsations is significantly higher because of the increase in the homogeneous linewidth relative to the Doppler linewidth. All these theoretical results are confirmed qualitatively in the experimental data referenced above.

\section{DISCUSSION}

One of the most intriguing aspects of laser oscillation is the occasional occurrence of spontaneous undamped output pulsations, especially when there are no obvious modulation mechanisms. The idea that there might be fundamental instabilities in the laser equations is as old as the invention of the laser. The particular type of instability discussed here can have a very low threshold and explains the spontaneous pulsations that have been observed in xenon lasers. More recently, similar pulsations have been obtained with the $3.39-\mu \mathrm{m}$ transition in helium-neon lasers. ${ }^{32}$ Although our original numerical solutions yielded qualitative agreement with observed experimental data, there were also some substantial quantitative discrepancies. The purpose of this study has been to develop an improved ring-laser model that takes account of the most important physical properties of a typical gas-laser system.

There are several respects in which this model has been improved. It now includes the possibility of direct spontaneous relaxation between the two laser states as well as the possibility of spectral cross relaxation due to velocity-changing collisions. Also, an attempt has been made to extract the most accurate physical parameters from the published xenon literature. As nearly as can be determined, the model seems to reproduce exactly the spontaneous-pulsation data obtained in our own xenon-laser experiments except for data that depend explicitly on the standing-wave nature of the fields for tuning near the Lamb dip. While it is harder to compare the model with data obtained elsewhere, our calculations for higher-pressure xenon lasers seem to be in general agreement with the experimental results of Maeda and Abraham ${ }^{24}$ and Abraham et al. ${ }^{12}$ It will be of interest in the future to use this model in exploring the actual or potential pulsation characteristics of other laser systems, and the results become especially important for applications that would benefit from the dramatically increased peak and average power.

\footnotetext{
* The author is also with the Department of Electrical Engineering, University of California, Los Angeles, California 90024.
}

\section{REFERENCES}

1. A. G. Gurtovnik, Izv. Vyssh. Uchebn. Zaved. Radiofiz. 1, 83 (1958).

2. A. V. Uspenskii, Radio Eng. Electron. Phys. 8, 1145 (1963).

3. V. V. Korobkin and A. V. Uspenskii, Sov. Phys. JETP 18, 693 (1964).

4. A. V. Uspenskii, Radio Eng. Electron. Phys. 9, 605 (1964).

5. A. Z. Grasyuk and A. N. Oraevskii, Radio Eng. Electron. Phys. 9, 424 (1964).

6. A. Z. Grasyuk and A. N. Oraevskii, in Quantum Electronics and Coherent Light, P. A. Miles, ed. (Academic, New York, 1964), p. 192.

7. See, for example, E. M. Belenov, V. N. Morozov, and A. N. Oraevskii in Quantum Electronics in Lasers and Masers, Part 2, D. V. Skolbel'tsyn, ed. (Plenum, New York, 1972), p. 217.

8. L. W. Casperson, in Third New Zealand Symposium on Laser Physics, Vol. 182 of Springer Lecture Notes in Physics (Springer-Verlag, Berlin, 1983), p. 88.

9. L. W. Casperson, "Modes and spectra of high gain lasers," Ph.D. dissertation (California Institute of Technology, 1971; University Microfilms, Ann Arbor, Mich., no. 72-469).

10. L. W. Casperson and A. Yariv, IEEE J. Quantum Electron. QE-8, 69 (1972).

11. L. W. Casperson, IEEE J. Quantum Electron. QE-14, 756 (1978).

12. N. B. Abraham et al., in Third New Zealand Symposium on Laser Physics, Vol. 182 of Springer Lecture Notes in Physics (Springer-Verlag, Berlin, 1983), p. 107.

13. H. Statz and G. DeMars, in Quantum Electronics, C. H. Townes, ed. (Columbia U. Press, New York, 1960), p. 530.

14. R. Dunsmuir, J. Electron. Control 10, 453 (1961).

15. G. Makhov, 'J. Appl. Phys. 33, 202 (1962).

16. D. M. Sinnett, J. Appl. Phys. 33, 1578 (1962).

17. L. W. Casperson, Phys. Rev. A 21, 911 (1980).

18. L. W. Casperson, Phys. Rev. A 23, 248 (1981).

19. L. W. Casperson, "Modes and spectra of high gain lasers," Ph.D. dissertation (California Institute of Technology, 1981; University Microfilms, Ann Arbor, Mich., no. 72-469), pp. 76 and 236.

20. J. Bentley and N. B. Abraham, Opt. Commun. 41, 52 (1982).

21. S. T. Hendow and M. Sargent III, Opt. Commun. 40, 385 (1982).

22. S. T. Hendow and M. Sargent III, Opt. Commun. 43, 59 (1982).

23. M. L. Minden and L. W. Casperson, IEEE J. Quantum Electron: QE-18, 1952 (1982).

24. M. Maeda and N. B. Abraham, Phys. Rev. A 26, 3395 (1982).

25. J. D. Gibbon and M. J. McGuinness, Physica 5D, 108 (1982).

26. P. Mandel, Opt. Commun. 44, 400 (1983).

27. P. Mandel, Opt. Commun. 45, 269 (1983).

28. N. B. Abraham, Laser Focus 19, 73 (1983).

29. R. Graham and Y. Cho, Opt. Commun. 47, 52 (1983).

30. P. Mandel and H. Zeghlache, Opt. Commun. 47, 146 (1983).

31. R. S. Gioggia and N. B. Abraham, Phys. Rev. Lett. 51, 650 (1983).

32. R. S. Gioggia and N. B. Abraham, Opt. Commun. 47, 278 (1983).

33. W. E. Lamb, Jr., Phys. Rev. 134, 1429 (1964).

34. P. W. Smith and T. Hänsch, Phys. Rev. Lett. 26, 740 (1971).

35. P. W, Smith, IEEE J. Quantum Electron. QE-8, 704 (1972).

36. H. Granek, C. Freed, and H. A. Haus, IEEE J. Quantum Electron. QE-8, 404 (1972).

37. C. Freed and H. A. Haus, IEEE J. Quantum Electron. QE-9, 219 (1973).

38. E. R. Peressini and G. J. Linford, IEEE J. Quantum Electron. QE-4, 657 (1968).

39. P. Cahuzac, O. Robaux, and R. Vetter, J. Phys. B 9, 3165 (1976).

40. P. Cahuzac, E. Marie, O. Robaux, R. Vetter, and P. R. Berman, J. Phys. B 11, 645 (1978).

41. J.-L. Le Gouet, J. Phys. B 11, 3001 (1978).

42. See, for example, T. Yajima and H. Souma, Phys. Rev. A 17, 309 (1978).

43. E. I. Gordon, A. D. White, and J. D. Rigden, in Proceedings of the Symposium on Optical Masers (Polytechnic Institute of Brooklyn, New York, 1963). 
44. P. O. Clark, R. A. Huback, and J. Y. Wada, JPL Contract No. 950803, Final Report, April 1965.

45. L. Allen, D. G. C. Jones, and D. G. Schofield, J. Opt. Soc. Am. 59, 842 (1969).

46. X. Husson and M. Margerie, Opt. Commun. 5, 139 (1972).

47. M. Aymar, Ph.D. Thesis, University of Paris (1973).

48. R. Vetter and E. Marie, J. Phys. B 11, 2845 (1978).

49. W. L. Faust, R. A. McFarlane, C. K. N. Patel, and C. G. B. Garrett, Appl. Phys. Lett. 1, 85 (1962).

50. W. L. Faust and R. A. McFarlane, J. Appl. Phys. 35, 2010 (1964).
51. J. Nella, S. Y. Szeto, P. Rabinowitz, and J. T. La Tourrette, IEEE J. Quantum Electron. QE-12, 543 (1976).

52. D. R. Armstrong, IEEE J. Quantum Electron. QE-4, 968 (1968).

53. C. K. N. Patel, Phys. Rev. 131, 1582 (1963).

54. J. W. Klüver, J. Appl. Phys. 37, 2987 (1966).

55. A. Dienes, Phys. Rev. 174, 414 (1968).

56. C. L. Tang and H. Statz, J. Appl. Phys. 38, 2963 (1967).

57. S. E. Schwartz and P. L. Gordon, J. Appl. Phys. 40, 4441 (1969).

\section{Lee W. Casperson}

Lee W. Casperson was born in Portland, Oregon in 1944. He received the B.S. degree in physics from the Massachusetts Institute of Technology in 1966 and the M.S. and Ph.D. degrees in electrical engineering and physics from the California Institute of Technology in 1967 and 1971, respectively. Since 1971 he has mostly been with the School of Engineering and Applied Science at the University of California, Los Angeles, where he is a professor. In 1981 he was a visiting professor in the Department of Physics, University of Auckland, Auckland, New Zealand, and he is now in the Department of Electrical Engineering, Portland State University, Portland, Oregon. He is an author of more than 90 publications relating primarily to laser physics, light scattering, modulation, and waveguides, and he recently received the Centennial Medal of the Institute of Electrical and Electronics Engineers for his achievements. As relates to the subject of this special issue, his research includes the experimental discovery and theoretical explanation of the first semiclassical-laser instability. 